\title{
Transcutaneous Auricular Vagus Nerve Stimulation Protects Endotoxemic Rat from Lipopolysaccharide-Induced Inflammation
}

\author{
Yu Xue Zhao, Wei He, Xiang Hong Jing, Jun Ling Liu, Pei Jing Rong, \\ Hui Ben, Kun Liu, and Bing Zhu \\ Institute of Acupuncture and Moxibustion, China Academy of Chinese Medical Sciences, 16 Nanxiaojie Street, \\ Dongzhimen Nei, Beijing 100700, China \\ Correspondence should be addressed to Bing Zhu, zhubing@mail.cintcm.ac.cn
}

Received 21 August 2012; Revised 5 December 2012; Accepted 6 December 2012

Academic Editor: Ying Xia

Copyright (c) 2012 Yu Xue Zhao et al. This is an open access article distributed under the Creative Commons Attribution License, which permits unrestricted use, distribution, and reproduction in any medium, provided the original work is properly cited.

\begin{abstract}
Background. Transcutaneous auricular vagus nerve stimulation (ta-VNS) could evoke parasympathetic activities via activating the brainstem autonomic nuclei, similar to the effects that are produced after vagus nerve stimulation (VNS). VNS modulates immune function through activating the cholinergic anti-inflammatory pathway. Methods. VNS, ta-VNS, or transcutaneous electrical acupoint stimulation (TEAS) on ST36 was performed to modulate the inflammatory response. The concentration of serum proinflammatory cytokines and tissue NF-kappa B p65 (NF- $\kappa$ B p65) were detected in endotoxaemia affected anesthetized rats. Results. Similar to the effect of VNS, ta-VNS suppressed the serum proinflammatory cytokines levels, such as tumour necrosis factor-alpha (TNF- $\alpha$ ), interleukin-1 beta (IL-1 $\beta$ ), and interleukin-6 (IL-6) as well as NF-kappa B p65 expressions of lung tissues. ST36 stimulation also decreases LPS-induced high TNF- $\alpha$ level and NF- $\kappa$ B signal, but it did not restrain proinflammatory cytokine IL- $1 \beta$ and IL-6. Neither ta-VNS nor ST36 stimulation could suppress LPS-induced TNF- $\alpha$ and NF- $\kappa$ B after vagotomy or with $\alpha 7 \mathrm{nAChR}$ antagonist injection. Conclusions. The present paper demonstrated that ta-VNS could be utilized to suppress LPSinduced inflammatory responses via $\alpha 7 \mathrm{nAChR}$-mediated cholinergic anti-inflammatory pathway.
\end{abstract}

\section{Introduction}

Inflammation is a local, protective response to microbial invasion or injury, which must be fine-tuned and regulated precisely [1]. Several potent cytokines including tumour necrosis factor-alpha (TNF- $\alpha$ ), interleukin-1 beta (IL-1 $\beta$ ), interleukin-6 (IL-6), interleukin-10 (IL-10), and transforming growth factor-beta (TGF- $\beta$ ) are produced by activated macrophages and other immune cells, as necessary and sufficient mediators involved in local and systemic inflammation $[2,3]$. Overproduction of cytokines leads to systematic inflammation and tissue injury. If the overproduced cytokines spread into the bloodstream, dangerous inflammatory responses will be induced. In the past ten years, abundant studies have been focused on "the cholinergic anti-inflammatory pathway," namely, the efferent vagus nerve which inhibits proinflammatory cytokine production and protects against systemic inflammation via a $\alpha 7 \mathrm{nAChR}$-dependent pathway [4]. Vagus nerve stimulation (VNS) prevents the occurrence and development of inflammation effectively via activating the cholinergic anti-inflammatory pathway. VNS and acetylcholine (Ach) attenuated the release of cytokines significantly and improved survival in lethal endotoxemia or sepsis models $[5,6]$. For instance, in a rat model of lethal endotoxamia, electrical stimulation of the efferent vagus nerve decreases serum and hepatic TNF levels [6]. Moreover, VNS inhibited all lipopolysaccharide- (LPS-) induced procoagulant responses strongly, attenuated the fibrinolytic response more modestly, and improved hepatic Ach levels significantly in endotoxemia rats [7]. VNS attenuated the LPS-induced increases of the plasma and splenic proinflammatory cytokines, rather than influencing the antiinflammatory cytokine IL-10 [8]. In addition, activation of this neural immune-modulatory pathway by electrical 
stimulation of vagus nerve also protects animals from various circumstances, such as ischemia-reperfusion injury, hypovolemic hemorrhagic shock, heart failure, and myocardial ischemia/reperfusion [9-12].

It was demonstrated that the transcutaneous auricular vagus nerve stimulation (ta-VNS) induced a series of parasympathetic activities [13-17]. Auricular branch of vagus nerve is a special vagal branch that innervates the body surface which could not be found on the other parts of the body [18], mainly innervating the cymba conchae and cavum conchae within the auricle. Our previous studies indicated that there is an intimate connection between auricular concha, the nucleus tractus solitarius (NTS), dorsal motor nucleus of the vagus nerve (DMN), and vagus nerve, which constructs the pathway of the auricular-vagal reflex. Accordingly, there might be some kinds of connection between auricular concha and efferent vagus nerve. In the present study, we reasoned that ta-VNS may have a role in activating the vagus nerve-based cholinergic anti-inflammatory pathway. Here, the effect of ta-VNS on proinflammatory cytokines and NF-kappa B p65 was explored to clarify the mechanism of taVNS underlying regulating inflammatory diseases.

\section{Materials and Methods}

2.1. Animals. Male Sprague Dawley rats ( 12 weeks old) were used in the present study, weighing $275-350 \mathrm{~g}$, supplied by China Academy of Military Science. Rats were housed in groups of 5-6 in standard polycarbonate cages at ambient temperature $\left(22^{\circ} \mathrm{C}\right)$ and allowed access to food and water $\mathrm{ad}$ libitum. Lights were set to an automated 07:00 on and 19:00 off light-dark cycle, and all animal experiments were done between 08:00 and 11:00 a.m. Rats received care consistent with the National Institutes of Health Guide for the Care and use of Laboratory Animals, and the experiments were conducted in accordance with a protocol approved by the Institutional Animal Care and Use Committee of China Academy of Chinese Medical Sciences.

2.2. Experimental Protocols. The present study consisted of 4 main parts. (1) The first detects the effect of ta-VNS, VNS, or transcutaneous electrical acupoint stimulation (TEAS) on ST36 on LPS-induced serum cytokine response. In this part, rats were randomly divided into 5 groups of twelve each: (a) saline-treated animals (NS), (b) endotoxemia model rats (LPS), (c) endotoxemia model rats receiving treatment of ta-VNS (ta-VNS), (d) endotoxemia model rats receiving treatment of VNS (VNS) and (e) endotoxemia model rats received treatment of TEAS on ST36 (ST36). (2) The second detects the effect of ta-VNS, VNS, or TEAS on ST36 on LPS-induced pulmonary NF- $\kappa \mathrm{B}$ p 65 expression. In this part, rats were randomly divided into 6 groups, which consisted of the 5 groups aforementioned, and a group of salinetreated animals received treatment of ta-VNS (NS+ta-VNS). (3) The third observes the effect after vagotomy (VGX). In this part, rats were randomly divided into 4 groups: (a) saline-treated animals (NS), (b) endotoxemia model rats (LPS), (c) ta-VNS-treated animals following vagotomy
(VGX+LPS+ta-VNS), and (d) TEAS-treated animals following vagotomy (VGX+LPS+ST36). (4) The fourth observes the effect after $\alpha 7 \mathrm{nAChR}$ antagonist injection. Rats were randomly divided into 4 groups: (a) saline-treated animals (NS), (b) endotoxemia model rats (LPS), (c) ta-VNS-treated animals following $\alpha$-bungarotoxin ( $\alpha$-BGT+LPS+ta-VNS), and (d) TEAS-treated animals following $\alpha$-bungarotoxin $(\alpha$ BGT+LPS+ST36).

2.3. Endotoxemia Model. LPS is an endotoxin derived from cell wall of gram-negative bacteria, and systemic injection of LPS results in various symptoms of bacterial infection including fever and inflammation [19]. Rats were injected intravenously with lipopolysaccharide (LPS, Escherichia coli 0111:B4; Sigma, $5 \mathrm{mg} / \mathrm{kg}$ ), dissolved in sterile, pyrogenfree saline that was sonicated for 30 minutes immediately before use. Rats ( $n=12$ per group) were killed 2 hours after LPS injection (Figure 1), and the blood was collected from abdominal aorta, allowed to clot for 2 hours at room temperature, and then centrifuged at room temperature for 15 minutes at $2000 \mathrm{rpm}$. Serum samples were stored at $-20^{\circ} \mathrm{C}$ before cytokine analysis. Lung samples were rapidly excised, rinsed of blood with normal saline, placed into liquid nitrogen immediately and then frozen and stored at $-80^{\circ} \mathrm{C}$ till measurement of NF- $\kappa \mathrm{B}$ p65 expression.

2.4. Electrical Vagus Nerve Stimulation. Rats were anaesthetized with urethane $(1 \mathrm{~g} / \mathrm{kg}$, intraperitoneally). A midline cervical incision was made to expose the left cervical branch of the vagus nerve. The left carotid sheath was isolated. After blunt preparation, the left vagus nerve trunk was carefully freed from surrounding tissue, separated from the carotid artery trunks, and placed on a custom-made bipolar platinum electrode connected via an isolation unit to a stimulator (SEN-7203, Nihon Kohden). All the exposed nerves were protected from dehydration by covering warm paraffin mineral oil tampons [6]. One and a half hour after LPS administration, constant electrical current stimuli with parameter of $1 \mathrm{~mA}, 10 \mathrm{~Hz}, 1 \mathrm{~ms}$ were turned on for $20 \mathrm{~min}$ (Figure 1(a)).

2.5. Transcutaneous Electrical Acupoint Stimulation (TEAS) on ST36. Transcutaneous surface electrodes were placed bilaterally on the depilatory rat skin at Zusanli (ST36). The ST36 points are located at $5 \mathrm{~mm}$ lateral to the anterior tubercle of the tibia and $10 \mathrm{~mm}$ below the knee joints. Bilateral surface electrodes at hind limbs were connected via an isolation unit to a stimulator (SEN-7203, Nihon Kohden), and the points were stimulated with the same parameter aforementioned. One and a half hour after LPS administration, the stimulation started and lasted for 20 min (Figure 1). Stimulation intensity was adjusted to a level that elicited a slight muscle twitch at the stimulated site and was limited to a maximum of $1 \mathrm{~mA}$ to minimize animal discomfort.

2.6. Transcutaneous Auricular Vagus Nerve Stimulation (taVNS). Transcutaneous surface electrodes were placed bilaterally on the auricular concha, which mainly includes cymba conchae and cavum conchae of the auricular. Bilateral surface electrodes at auricular concha were connected via an 


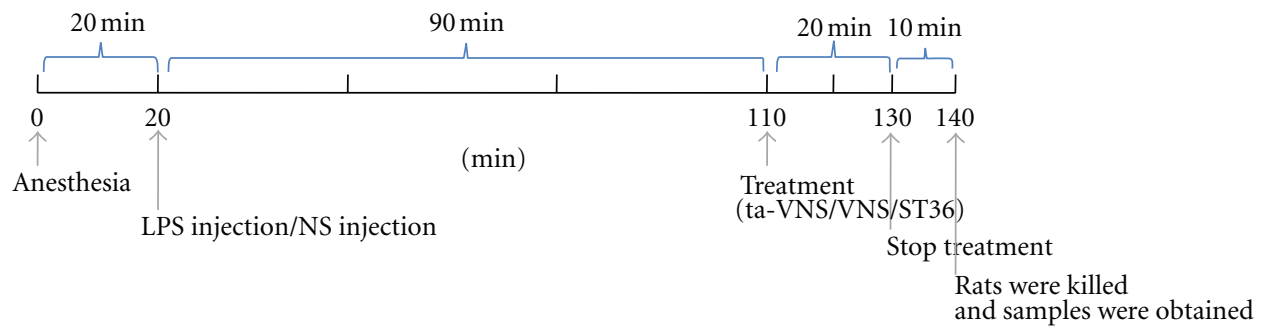

(a)

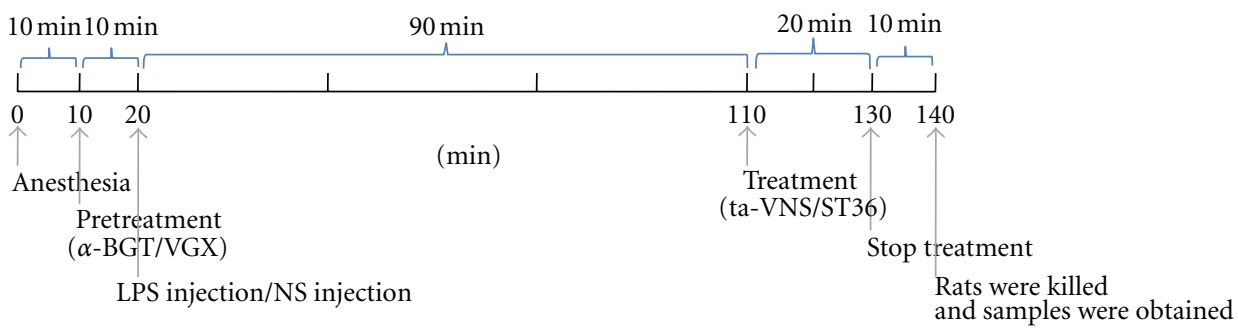

(b)

FIGURE 1: The time flow chart indicates the precise time for various operations in the present study, from the time point of anesthetic injection to the time point for sampling. (a) Twenty minutes after anesthesia, rats were injected intravenously with LPS or NS. One and a half hour after modeling, treatment (ta-VNS, VNS, or TEAS on ST36) was performed for twenty minutes. Two hours after LPS injection, rats were killed, and samples were collected. (b) Ten minutes after anesthesia, administration of $\alpha$-BGT or vagotomy was performed. The rest of the operations were the same with the time flow in (a).

isolation unit to a stimulator (SEN-7203, Nihon Kohden), and the auricular conchae on both sides were stimulated with the same parameter. One and a half hour after LPS administration, the stimulation started and lasted for $20 \mathrm{~min}$ (Figure 1). Stimulation intensity was adjusted to a level that elicited a slight twitch of the auricle and was limited to maximum of $1 \mathrm{~mA}$ to minimize animal discomfort.

2.7. Vagotomy. Vagotomy was performed before LPS administration (Figure 1(b)). In vagotomized animals, following a ventral cervical midline incision, bilateral vagus trunks were exposed and separated from the common carotid artery, ligated with a 4-0 silk suture.

2.8. Administration of $\alpha 7 n A C h R$ Antagonist. The specific $\alpha 7 \mathrm{nAChR}$ antagonist $\alpha$-bungarotoxin $(\alpha$-BGT) was obtained from Alexis Biochemicals Corporation (San Diego, CA, USA). The drug was administered intravenously at a dose of $1 \mu \mathrm{g} / \mathrm{kg}$ before LPS administration [20] (Figure 1(b)).

2.9. Cytokine Analysis. Abdominal aortic blood was collected two hours after LPS administration, allowed to clot for $2 \mathrm{~h}$ at room temperature, and centrifuged for $20 \mathrm{~min}$ at $2500 \mathrm{rpm}$. Serum TNF- $\alpha$, IL- $1 \beta$, and IL- 6 concentrations were analyzed, respectively, by TNF- $\alpha$, IL- $1 \beta$, and IL-6 ELISA kits (R\&D Systems) following the manufacturer's instructions.

2.10. Western Blot. Procedures of western blot analysis were followed as described previously [21]. Protein samples denatured in SDS sample buffer $(125 \mathrm{mmol} / \mathrm{L}$ Tris- $\mathrm{HCl}$, pH 6.8, 50\% glycerol, 2\% SDS, 5\% mercaptoethanol, and $0.01 \%$ bromophenol blue) were subjected to SDS-PAGE and blotted onto Immobilon-FL transfer membrane (Millipore). Blotted membranes were blocked with $5 \%$ skim milk in Trisbuffered saline containing $0.05 \%$ Tween-20 for 2 hours and were subsequently incubated with rabbit anti-human-NF- $\kappa \mathrm{B}$ (p65) (diluted 1/200; Santa Cruz Biotechnology Inc., CA, USA) overnight at $4^{\circ} \mathrm{C}$. After three washes in Tris-buffered saline containing $0.05 \%$ Tween 20 , the membranes were incubated with an anti-rabbit IgG antibody-HRP (diluted 1/4000; Santa Cruz Biotechnology Inc., CA, USA) for 1 hour. Quantification of western blots was performed by the Odyssey infrared imaging system (Li-Cor Biosciences) to detect protein expression.

2.11. NF- $\kappa B$ Immune-Histochemistry. NF- $\kappa \mathrm{B}$ p65 immunohistochemistry staining was performed as described previously [22] to evaluate the lung tissues inflammatory response. Briefly, tissue sections were deparaffinized with xylene and rehydrated through graded series of alcohols. Tissue sections were rinsed in PBS, pretreated with citrate buffer at $93^{\circ} \mathrm{C}$, blocked with PBS containing $2 \%$ BSA, and then incubated with a primary antibody reactive against rabbitactivated p65 subunit of NF- $\kappa$ B (Santa Cruz Biotechnology Inc, Santa Cruz, CA, USA). Washed sections were incubated for $10 \mathrm{~min}$ with secondary goat anti-rabbit IgG biotin. The reaction product was visualized with $\mathrm{DAB}$ chromogenic agent. The sections were counterstained with hematoxylin stain. Slides were analysed on a light microscope (Olympus BX60) using an ImagePro Plus Imaging System (Universal Imaging).

2.12. Statistical Analysis. All the data in the present study were expressed as means \pm SEM and analyzed by one-way 


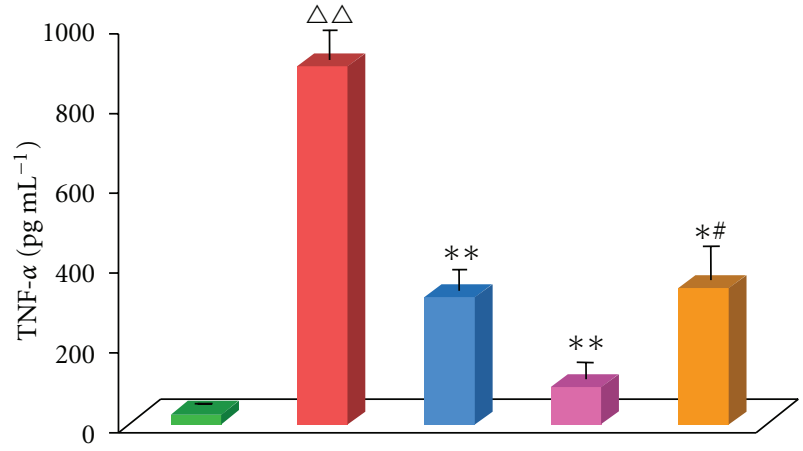

(a)

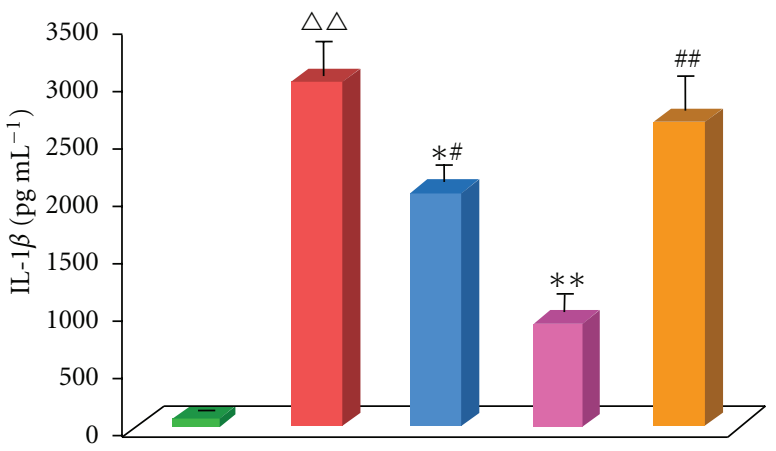

(b)

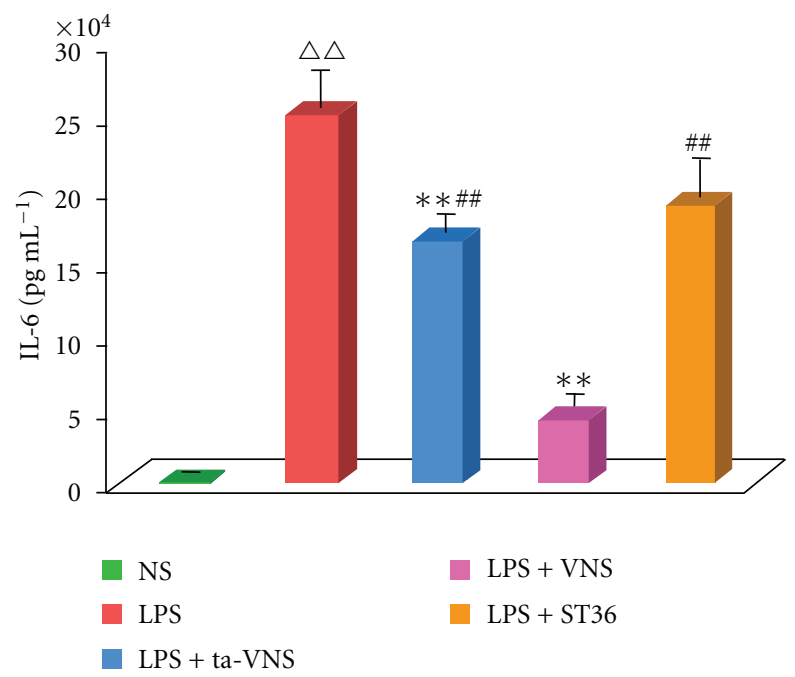

(c)

FIGURE 2: Vagus nerve stimulation (VNS) or transcutaneous auricular vagus nerve stimulation (ta-VNS) attenuates the LPS-induced serum cytokine (TNF- $\alpha$, IL- $1 \beta$, and IL-6) response. TEAS on ST36 inhibited TNF- $\alpha$ level significantly. Serum TNF- $\alpha$ (a), IL- $1 \beta$ (b), and IL-6 (c) contents were measured by ELISA. The columns represent mean \pm SEM for 12 animals in each group. ${ }^{\Delta} P<0.01$ versus the normal saline (NS) group; ${ }^{*} P<0.05$ versus LPS group (LPS); ${ }^{* *} P<0.01$ versus LPS group (LPS); ${ }^{*} P<0.05$ versus LPS+VNS group; ${ }^{\# \# ~} P<0.01$ versus LPS+VNS group.

ANOVA with SPSS software. The two-tailed Student's $t$ test was used to compare mean values between two groups. $P$ values $<0.05$ were considered significant.

\section{Results}

3.1. Cytokine Levels in the Serum. LPS evoked an inflammatory response characterized by the upregulation of cytokine expressions. After systemic administration of LPS $(5 \mathrm{mg} / \mathrm{kg}$, i.v.), TNF- $\alpha$ (Figure $2(\mathrm{a})$ ), IL-1 $\beta$ (Figure $2(\mathrm{~b})$ ), and IL-6 (Figure 2(c)) increased significantly in sera. Both electrical VNS and ta-VNS strongly inhibited LPS-induced proinflammatory cytokine concentrations including TNF- $\alpha$, IL- $1 \beta$, and IL-6 $(n=12, P<0.01, P<0.05$, resp.). TEAS of ST36 lowered serum TNF- $\alpha$ level $(n=12, P<0.05)$ in endotoxemic rats but failed to significantly alter serum IL- $1 \beta$ and serum IL-6 levels.

3.2. The Effect of ta-VNS or TEAS on ST36 on Serum TNF$\alpha$ Level Was Blocked by $\alpha-B G T$ Administration. The above results showed that ta-VNS has similar effects to VNS on cytokine levels. Previous studies show that VNS-activated "cholinergic anti-inflammatory pathway" regulates systemic inflammatory responses via $\alpha 7 \mathrm{nAChR}$, hereby ta-VNS may have the same effect. To test this hypothesis, we pretreated animals with the $\alpha 7 \mathrm{nAChR}$ antagonist $\alpha$-BGT. LPS injection induced profound rise in the concentration of serum TNF- $\alpha$. Either ta-VNS or TEAS on ST36 failed to inhibit TNF- $\alpha$ level after $\alpha$-BGT administration (Figure 3).

3.3. Effect of ta-VNS or TEAS on ST36 on Serum TNF- $\alpha$ Was Blunted by Vagotomy. To examine the mechanism of ta-VNS in "cholinergic anti-inflammatory pathway," we pretreated animals with vagotomy. The result indicated that intravenous injection of LPS elicited a rapid raise of TNF- $\alpha$ level. Neither ta-VNS nor TEAS on ST36 was effective on inhibiting TNF- $\alpha$ level after vagotomy (Figure 4).

3.4. NF-Kappa B p65 Expressions in Lung Tissues. The systemic administration of LPS was followed with a significantly 


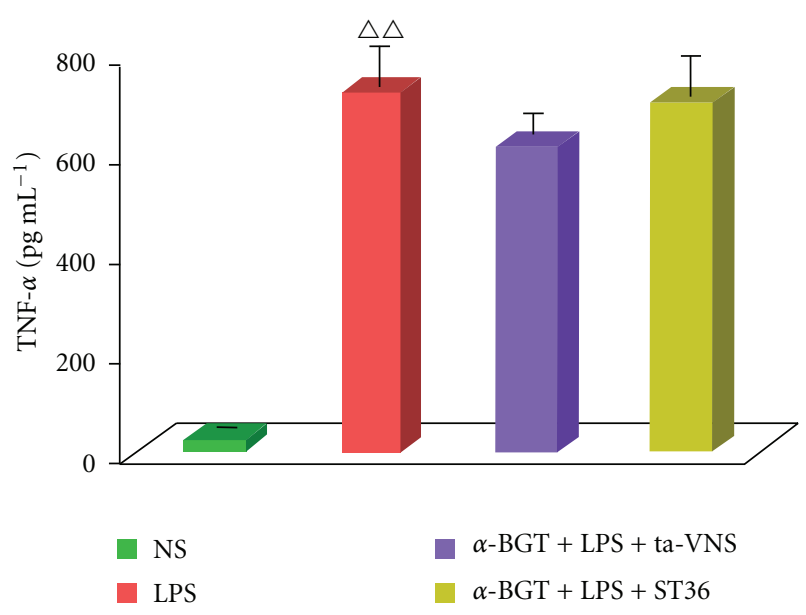

FIgURE 3: ta-VNS or TEAS on ST36 with $\alpha$-bungarotoxin $(\alpha$ BGT) administration fails to inhibit the LPS-induced serum TNF- $\alpha$ response. Serum TNF- $\alpha$ concentrations were measured by ELISA. Data are expressed as mean $\pm \operatorname{SEM}\left(n=12\right.$ per group). ${ }^{\triangle} P P<0.01$ versus the normal saline (NS) group.

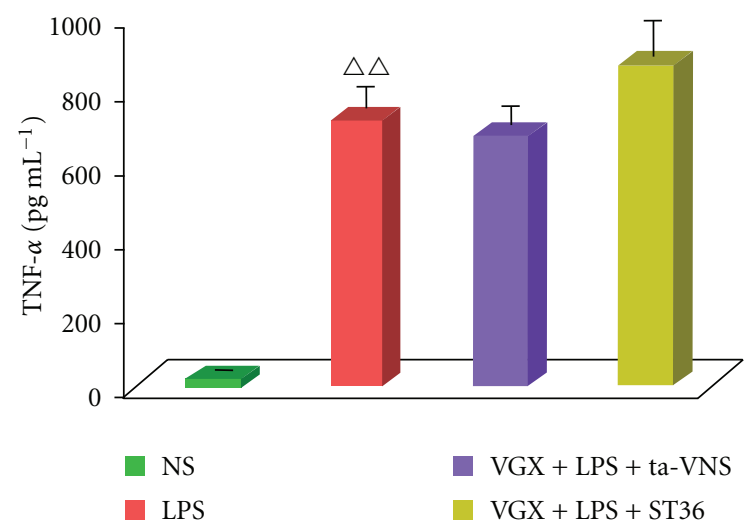

FIGURE 4: ta-VNS or ST36 stimulation with bilateral cervical vagotomy (VGX) fails to inhibit the LPS-induced serum TNF$\alpha$ response. TNF- $\alpha$ amounts were measured by ELISA. Data are expressed as mean $\pm \operatorname{SEM}$ ( $n=12$ per group). ${ }^{\Delta} P<0.01$ versus the normal saline (NS) group.

increased expression of NF- $\kappa \mathrm{B}$ p65 in lung tissues (Figures 4 and 5). Both electrical VNS and ta-VNS strongly inhibited LPS-induced NF- $\kappa \mathrm{B}$ p 65 ( $n=10, P<0.01$, Figures 4 and 5$)$. TEAS on ST36 did not have the same effect (Figures 5 and 6).

3.5. Effect of ta-VNS or TEAS on ST36 on NF- $\kappa B$ p 65 Was Blunted by Vagotomy. We pretreated animals with vagotomy. The result indicated that ta-VNS or ST36 failed to inhibit the expressions of NF- $\kappa$ B p65 after vagotomy (Figure 7 ).

\section{Discussion}

Here, we reported our original study that auricular concha stimulation is also a potent anti-inflammatory stimulus that can modulate immune factors in endotoxemia rat model. The present study demonstrates that ta-VNS may have an
$\mathrm{NF}-\kappa \mathrm{B}$ p65 (65kDa)

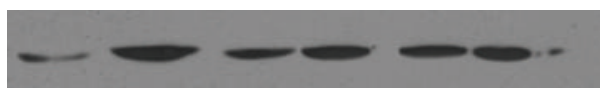

$\operatorname{Actin}(43 \mathrm{kDa})$
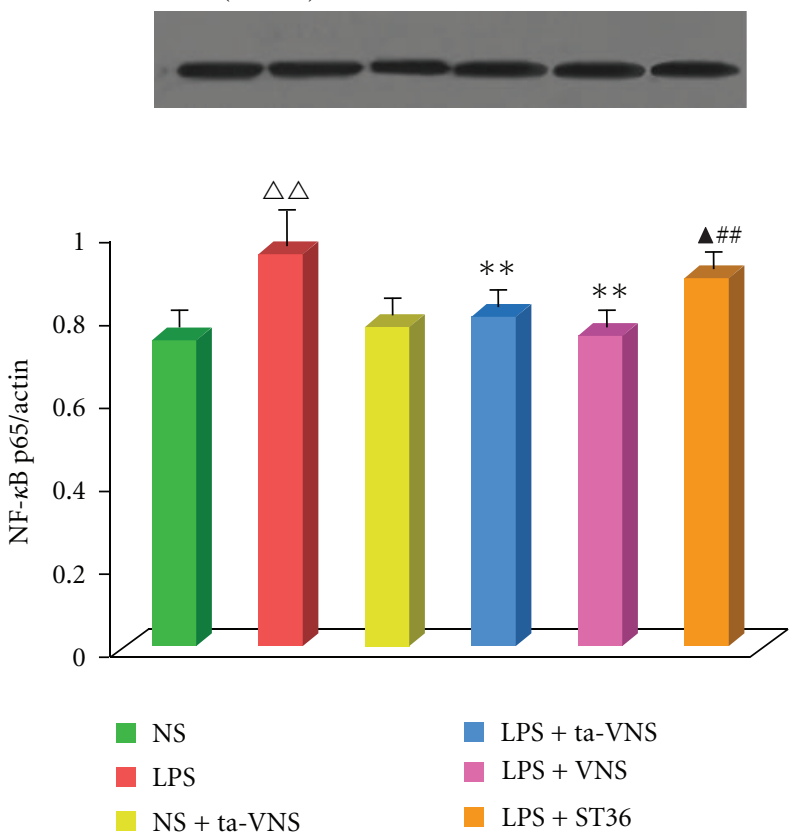

FIGURE 5: VNS or ta-VNS suppresses LPS-induced NF- $\kappa$ B expression; ST36 stimulation did not affect NF- $\kappa \mathrm{B}$ in endotoxemia animals significantly. ta-VNS did not significantly affect pulmonary $\mathrm{NF}-\kappa \mathrm{B}$ expression with normal saline administration. NF- $\kappa \mathrm{B}$ expressions were measured by western blot technique. Data are shown by mean \pm SEM ( $n=12$ per group). ${ }^{\Delta \Delta} P<0.01$ versus normal saline (NS) group; ${ }^{* *} P<0.01$ versus LPS group (LPS); ${ }^{\#} P<0.01$ versus LPS+VNS group; ${ }^{\wedge} P<0.05$ versus LPS+ ta-VNS.

important role in suppressing inflammatory responses, and this contributes to the involvement of the cholinergic antiinflammatory pathway in the mechanism.

Previous study demonstrated that the cholinergic antiinflammatory pathway is a $\alpha 7 \mathrm{nAChR}$-dependent, vagus nerve-mediated pathway [1]. It can inhibit macrophage activation through parasympathetic outflow, which functions as an anti-inflammatory pathway in systemic and local inflammation. Inflammatory signals stimulate sensory fibers that ascend in the vagus nerve to synapse in the NTS and then activate efferent fibers in the vagus nerve to suppress peripheral cytokine release through alpha7nAChR.

The most important cytokine involved is TNF- $\alpha$, which activates other proinflammatory cytokines such as IL- $1 \beta$, IL-6, and high mobility group B1 (HMGB1) and amplifies other inflammatory mediators. VNS has been demonstrated to inhibit proinflammatory cytokine production [23-25], especially the release and synthesis of TNF- $\alpha$. The present study indicates that VNS decreases LPS-induced TNF- $\alpha$, IL-1, and IL-6 in circulation. And ta-VNS reduced the levels of proinflammatory cytokines TNF- $\alpha$, IL- $1 \beta$, and IL6 , which is similar to the effect of VNS. After administration of $\alpha 7 \mathrm{nAChR}$ antagonist $\alpha$-BGT, ta-VNS failed to attenuate 
NS

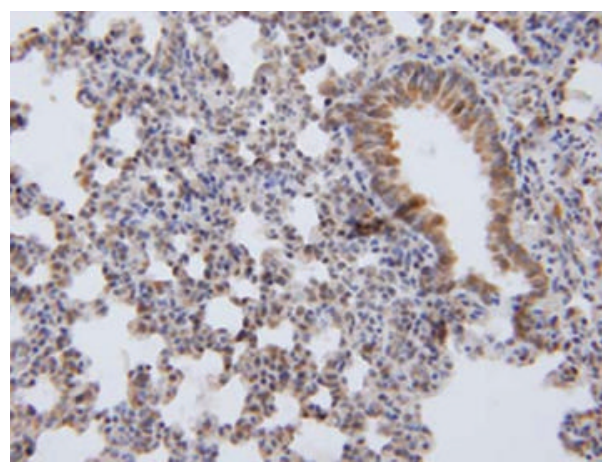

(a)

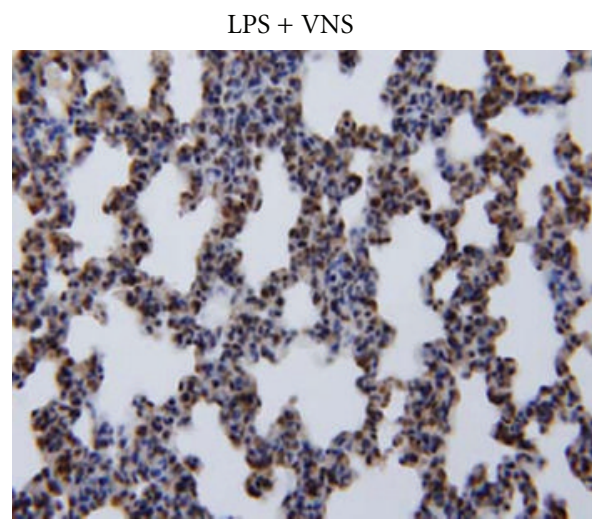

(c)

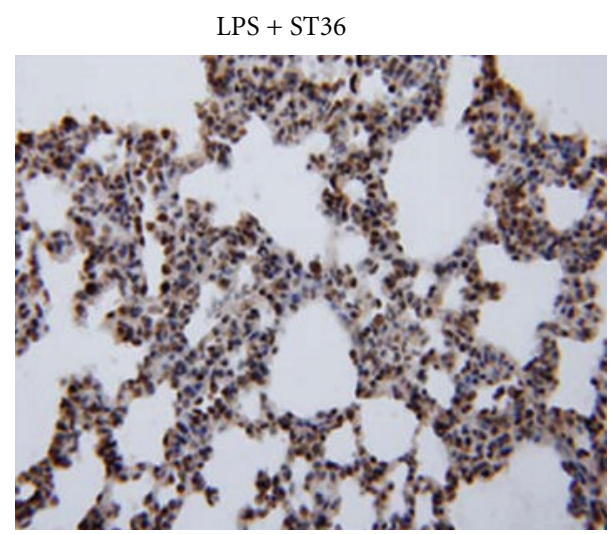

(e)
LPS

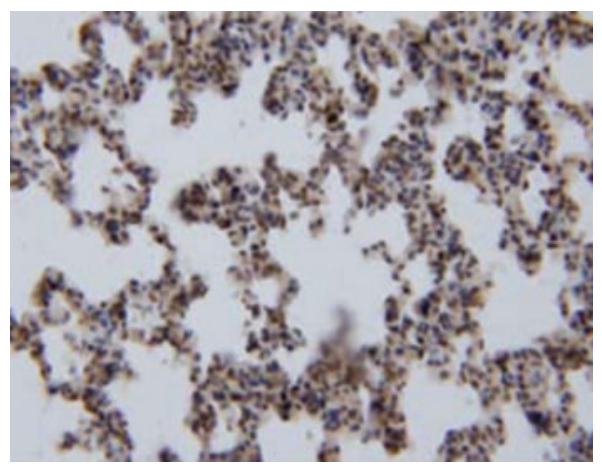

(b)

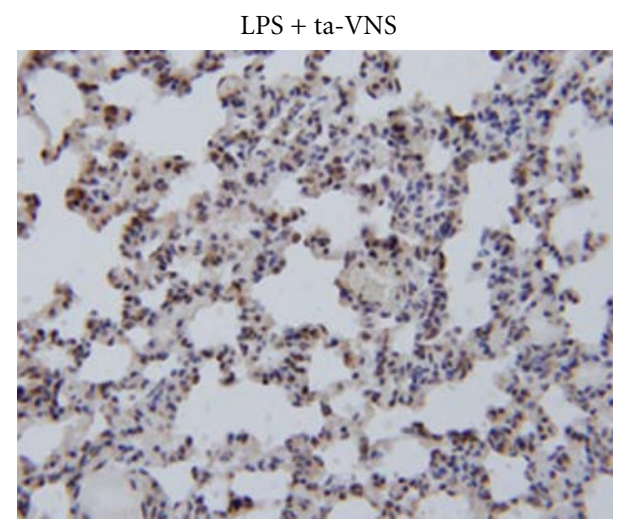

(d)

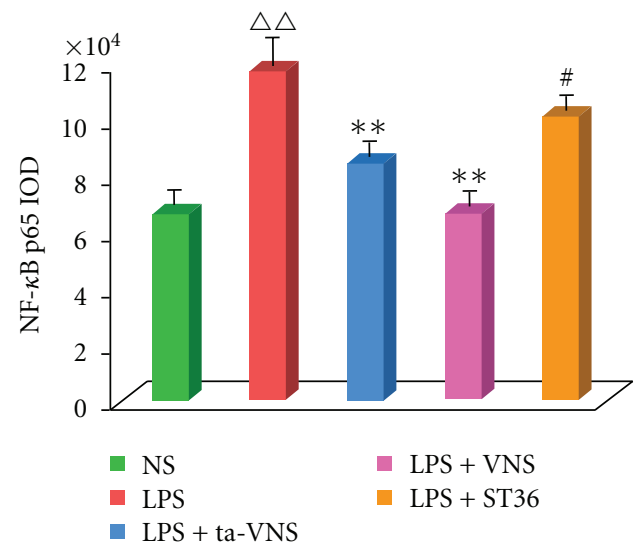

(f)

FIGURE 6: Immunohistochemical staining with anti-NF- $\kappa$ B antibodies reveals significant decrease in LPS-induced NF- $\kappa$ B immunoreactivity evoked by interventions as of VNS, ta-VNS, and TEAS on ST36. Data are expressed as mean \pm SEM $(n=12$ per group). $\Delta \Delta P<0.01$ versus the normal saline (NS) group; ${ }^{* *} P<0.01$ versus LPS group (LPS); ${ }^{*} P<0.05$ versus LPS+VNS group. Original magnification: $\times 400$.

serum TNF- $\alpha$ level, which is consistent with previous reports $[6-10,20,23]$. This result indicated that $\alpha 7 \mathrm{nAChR}$ played a critical role in anti-inflammatory effect of ta-VNS. The present study also demonstrated that vagotomy exacerbated serum TNF responses to inflammatory stimulation, sensitized animals to the lethal effects of endotoxin, and abolished the anti-inflammatory effect of ta-VNS. The results indicated that ta-VNS fails to suppress excessive cytokine response characterized by exaggerated TNF- $\alpha$ level if there is deficiency in either the $\alpha 7 \mathrm{nAChR}$ subunit or vagus nerve.

$\mathrm{NF}-\kappa \mathrm{B}$ is a master transcription factor controlling the expression of a wide range of proinflammatory genes [2628]. Previous studies reported that NF- $\kappa \mathrm{B}$ is involved in TNF- $\alpha$ genetic activation and TNF- $\alpha$ production $[29,30]$. In the present study, both western blot data and immunehistochemical results indicated that ta-VNS suppresses the 
NS

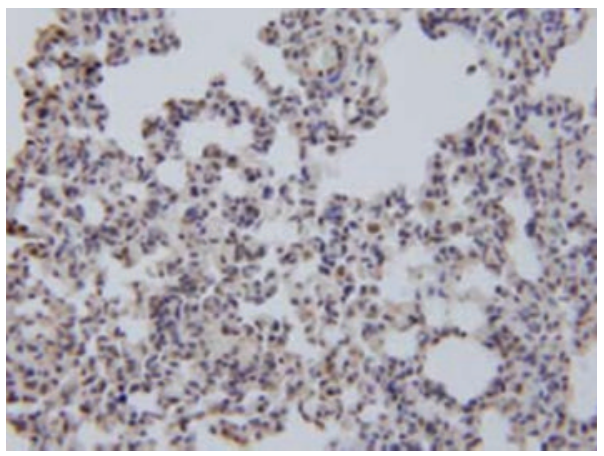

(a)

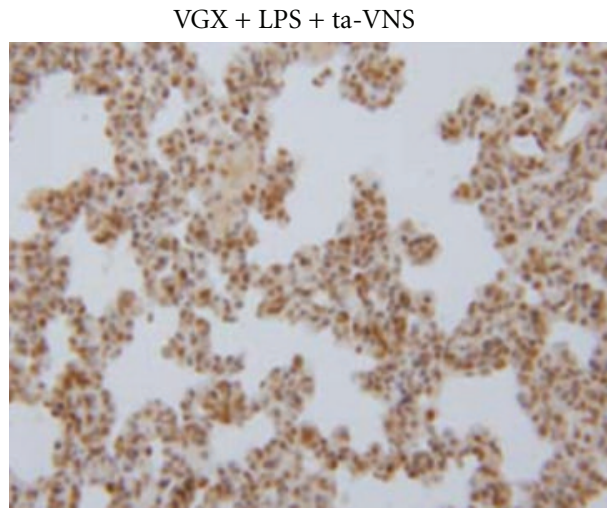

(c)
LPS

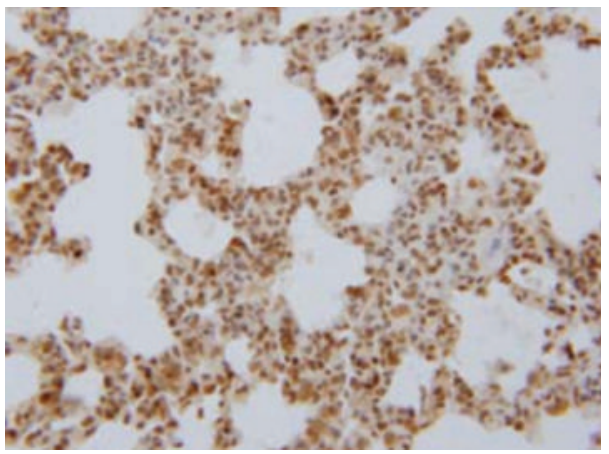

(b)

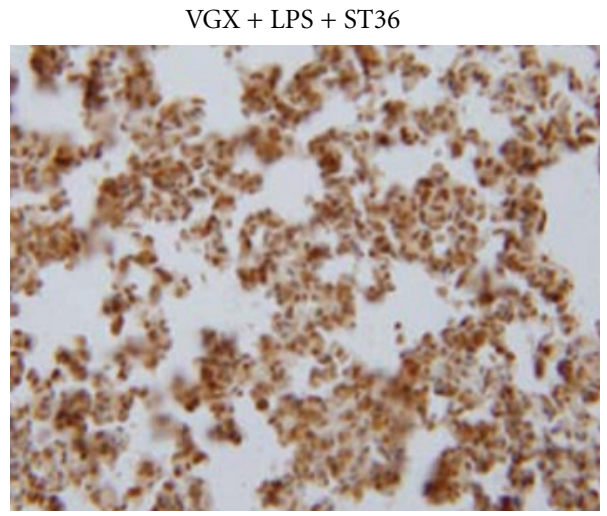

(d)

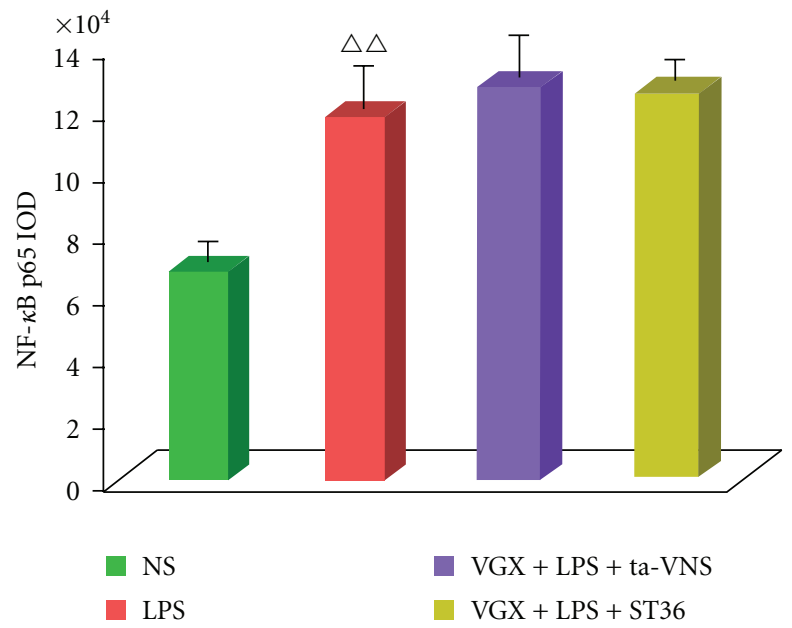

(e)

FIGURE 7: ta-VNS or ST36 stimulation with bilateral cervical vagotomy (VGX) fails to inhibit the LPS-induced overexpression of NF- $\kappa$ B. $\mathrm{NF}-\kappa \mathrm{B}$ distribution was measured by immunohistochemical staining. Data are shown as mean $\pm \operatorname{SEM}\left(n=12\right.$ per group). ${ }^{\Delta} P<0.01$ versus the normal saline (NS) group. Original magnification: $\times 400$.

LPS-induced NF- $\kappa \mathrm{B}$ expression in rat lung tissue (Figures 4 and 5), which mimicking the effects of VNS [25, 31]. In vagotomy animals, ta-VNS failed to inhibit increased NF- $\kappa \mathrm{B}$ expression, suggesting ta-VNS functions in situations that require intact vagus nerve.

Some investigators demonstrated that electroacupuncture (EA, on ST36, PC6, and GV20) could increase the vagal activity of experimental animals and human subjects
[13-17, 32-34]. The present study demonstrated that TEAS on ST36 decreased serum TNF- $\alpha$ level in endotoxemia rats. After pretreatment with vagotomy or $\alpha 7 \mathrm{nAChR}$ antagonist $\alpha$-BGT, TEAS failed to inhibit serum proinflammatory level in LPS-induced endotoxemia animals.

Auricular acupuncture, as a special form of acupuncture, has been used for the treatment of different disorders for centuries in China. Our research group previously demonstrated 

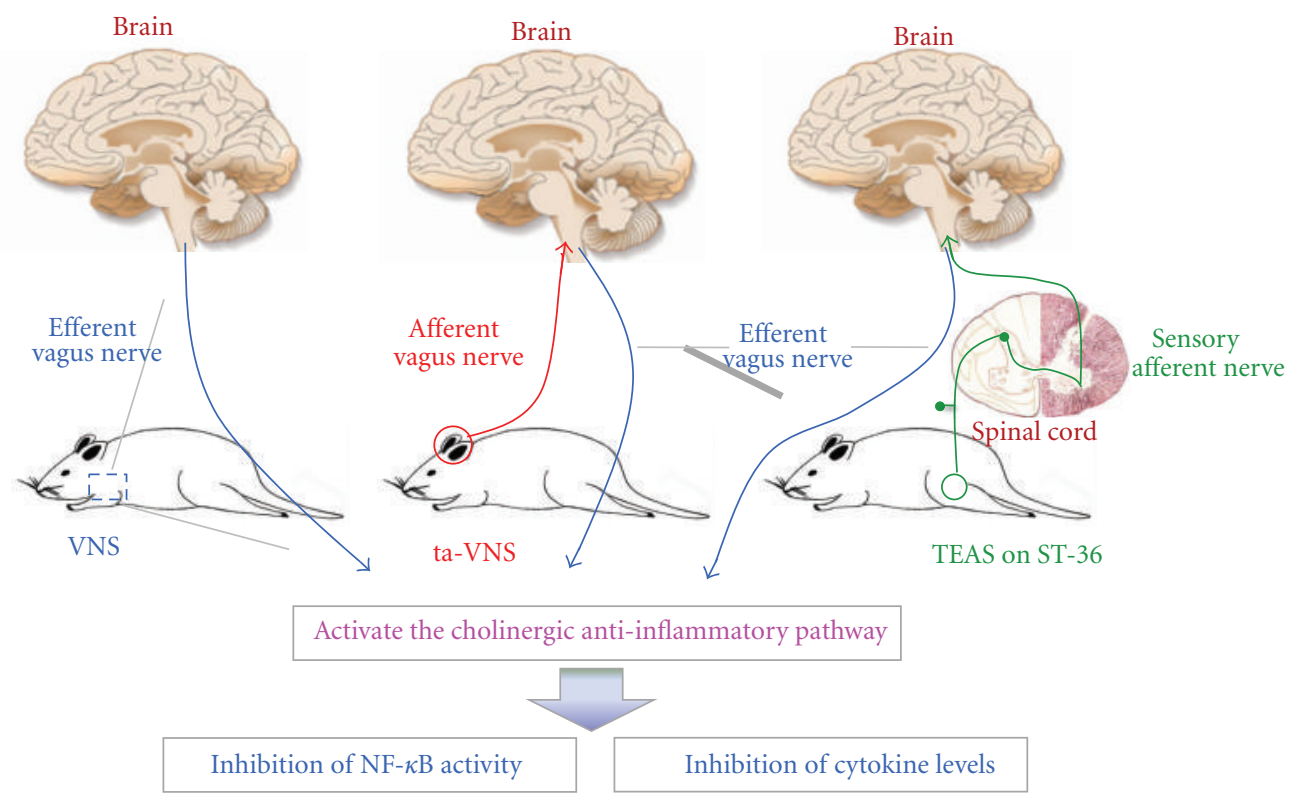

FIGURE 8: The anti-inflammatory mechanisms of the three interventions used in the present study might be as follows: (1) VNS directly activates the cholinergic anti-inflammatory pathway via stimulating efferent vagus nerve. (2) ta-VNS evoked the activity of the auricular branch of vagus nerve $(\mathrm{ABVN})$. The activated signals ascending within afferent vagus nerve are transmitted to the nucleus tractus solitarius. The integrated output is carried by the efferent vagus nerve to inhibit inflammatory responses. (3) TEAS on ST36 activates the somatic fiber endings of the skin around ST36 point, sending signals to the spinal cord via somatic sensory nerve fibers. The nerve impulses were relayed and integrated by NTS by the secondary order neurons in the spinal cord, and the cholinergic anti-inflammatory pathways are activated by the increased efferent vagal output.

that auricular acupuncture stimulation could activate neurons of NTS and upregulate vagal tune, to decrease MAP and HR [35], to trigger gastric motility [36]. Our previous studies also demonstrated that TEAS of auricular concha could activate the parasympathetic nervous system and mimic the effect of VNS to suppress epileptic seizures [18]. In the present study, the results showed that ta-VNS inhibited proinflammatory cytokine levels and suppressed NF- $\kappa$ B expressions in endotoxaemia rats (Figures 1,4 , and 5 ), which is similar to the effect of VNS. However, vagotomy or $\alpha 7 \mathrm{nAChR}$ antagonist $\alpha$-BGT could diminish the effect of ta-VNS on the anti-inflammatory responses, suggesting that auricular acupuncture may perform an anti-inflammatory effect via cholinergic anti-inflammatory pathway.

In general (Figure 8 ), VNS directly activates the cholinergic anti-inflammatory pathway via stimulating efferent vagus nerve. As the peripheral branch [18], auricular branch of vagus nerve (ABVN) innervates the auricular concha and the external auditory meatus. Stimulation of the ABVN region could evoke parasympathetic excitation [37-39]. Acupuncture in the area of auricular concha may increase discharge of NTS [18], as the central terminal nuclear for afferent vagal fibers, which primarily transmit signals from local inflammation lesion $[4,40]$. Thus, we hypothesize that ABVN could be evoked by ta-VNS, and the activated signals ascend with vagal input to the NTS. The signals are processed within the NTS, and the integrated output signal is carried by efferent vagus nerve to inhibit inflammatory responses. TEAS on the acupoint of ST36 activates the somatic fiber endings around ST36 point, which send the acupuncture signals to the spinal cord via somatic sensory nerve fibers. In the spinal cord, the nerve impulses are delivered to the NTS by the secondary order neurons, where the signals were processed. Ultimately, the increased efferent vagal output activates the cholinergic anti-inflammatory pathway.

\section{Conclusions}

The results presented here demonstrate that ta-VNS plays an important role in immuneregulation, through the activation of the cholinergic anti-inflammatory pathway and the downregulation of proinflammatory cytokine expressions and NF$\kappa \mathrm{B}$ activities. VNS and TEAS on ST36 might suppress the inflammatory responses via different mechanisms.

\section{Abbreviations}

ta-VNS: Transcutaneous auricular vagus nerve stimulation

LPS: lipopolysaccharide

NTS: $\quad$ Nucleus tractus solitarius

DMN: Dorsal motor nucleus of the vagus

VNS: Vagus nerve stimulation

TEAS: Transcutaneous electric acupoint stimulation

Ach: Acetylcholine

$\alpha 7 n A C h R:$ Nicotinic acetylcholine receptors

NF- $\kappa$ B p65: NF-kappa B p65

TNF- $\alpha$ : Tumour necrosis factor-alpha

IL-1 $\beta$ : Interleukin- 1 beta 
IL-6: Interleukin-6

$\alpha$-BGT: $\alpha$-Bungarotoxin

NS: Normal saline

VGX: Vagotomy

ABVN: Auricular branch of vagus nerve.

\section{Acknowledgments}

The authors thank Dr. Gao Xinyan for her excellent editorial assistance. They thank Dr. Qiao Haifa for his critical reading of the paper. This work was supported by the National Basic Research Program (973 Program, no. 2011CB505201), the National Natural Science Foundation of China Research Grants (nos. 30973798 and 30901931), and the Beijing Natural Science Foundation (no. 7111007).

\section{References}

[1] K. J. Tracey, “The inflammatory reflex,” Nature, vol. 420, no. 6917, pp. 853-859, 2002.

[2] K. J. Tracey, Y. Fong, D. G. Hesse et al., "Anti-cachectin/TNF monoclonal antibodies prevent septic shock during lethal bacteraemia," Nature, vol. 330, no. 6149, pp. 662-664, 1987.

[3] K. J. Tracey, B. Beutler, and S. F. Lowry, "Shock and tissue injury induced by recombinant human cachectin," Science, vol. 234, no. 4775, pp. 470-474, 1986.

[4] V. A. Pavlov and K. J. Tracey, "The cholinergic anti-inflammatory pathway," Brain, Behavior, and Immunity, vol. 19, no. 6, pp. 493-499, 2005.

[5] J. M. Huston, M. Gallowitsch-Puerta, M. Ochani et al., "Transcutaneous vagus nerve stimulation reduces serum high mobility group box 1 levels and improves survival in murine sepsis," Critical Care Medicine, vol. 35, no. 12, pp. 2762-2768, 2007.

[6] L. V. Borovikova, S. Ivanova, M. Zhang et al., "Vagus nerve stimulation attenuates the systemic inflammatory response to endotoxin," Nature, vol. 405, no. 6785, pp. 458-462, 2000.

[7] J. Huang, Y. Wang, D. Jiang, J. Zhou, and X. Huang, "The sympathetic-vagal balance against endotoxemia," Journal of Neural Transmission, vol. 117, no. 6, pp. 729-735, 2010.

[8] D. J. van Westerloo, I. A. J. Giebelen, J. C. M. Meijers et al., "Vagus nerve stimulation inhibits activation of coagulation and fibrinolysis during endotoxemia in rats," Journal of Thrombosis and Haemostasis, vol. 4, no. 9, pp. 1997-2002, 2006.

[9] T. R. Bernik, S. G. Friedman, M. Ochani et al., "Pharmacological stimulation of the cholinergic antiinflammatory pathway," Journal of Experimental Medicine, vol. 195, no. 6, pp. 781-788, 2002.

[10] S. Guarini, D. Altavilla, M. M. Cainazzo et al., "Efferent vagal fibre stimulation blunts nuclear factor- $\kappa$ B activation and protects against hypovolemic hemorrhagic shock," Circulation, vol. 107, no. 8, pp. 1189-1194, 2003.

[11] M. Li, C. Zheng, T. Sato, T. Kawada, M. Sugimachi, and K. Sunagawa, "Vagal nerve stimulation markedly improves longterm survival after chronic heart failure in rats," Circulation, vol. 109, no. 1, pp. 120-124, 2004.

[12] C. Mioni, C. Bazzani, D. Giuliani et al., "Activation of an efferent cholinergic pathway produces strong protection against myocardial ischemia/reperfusion injury in rats," Critical Care Medicine, vol. 33, no. 11, pp. 2621-2628, 2005.

[13] S. T. Huang, G. Y. Chen, H. M. Lo, J. G. Lin, Y. S. Lee, and C. D. Kuo, "Increase in the vagal modulation by acupuncture at Neiguan point in the healthy subjects," American Journal of Chinese Medicine, vol. 33, no. 1, pp. 157-164, 2005.

[14] Z. Li, K. Jiao, M. Chen, and C. Wang, "Effect of magnitopuncture on sympathetic and parasympathetic nerve activities in healthy driver-assessment by power spectrum analysis of heart rate variability," European Journal of Applied Physiology, vol. 88, no. 4-5, pp. 404-410, 2003.

[15] J. H. Wu, H. Y. Chen, Y. J. Chang et al., "Study of autonomic nervous activity of night shift workers treated with laser acupuncture," Photomedicine and Laser Surgery, vol. 27, no. 2, pp. 273-279, 2009.

[16] C. C. Hsu, C. S. Weng, T. S. Liu, Y. S. Tsai, and Y. H. Chang, "Effects of electrical acupuncture on acupoint BL15 evaluated in terms of heart rate variability, pulse rate variability and skin conductance response," American Journal of Chinese Medicine, vol. 34, no. 1, pp. 23-36, 2006.

[17] K. Imai and H. Kitakoji, "Comparison of transient heart rate reduction associated with acupuncture stimulation in supine and sitting subjects," Acupuncture in Medicine, vol. 21, no. 4, pp. 133-137, 2003.

[18] W. He, P. J. Rong, L. Li, H. Ben, B. Zhu, and G. Litscher, "Auricular acupuncture may suppress epileptic seizures via activating the parasympathetic nervous system: a hypothesis based on innovative methods," Evidence-Based Complementary and Alternative Medicine, vol. 2012, Article ID 615476, 5 pages, 2012.

[19] L. Bertók, "Endotoxins and endocrine system," Domestic Animal Endocrinology, vol. 15, no. 5, pp. 305-308, 1998.

[20] H. Wang, M. Yu, M. Ochani et al., "Nicotinic acetylcholine receptor $\alpha 7$ subunit is an essential regulator of inflammation," Nature, vol. 421, no. 6921, pp. 384-388, 2003.

[21] W. Deng, X. Wang, J. Xiao et al., "Loss of regulator of G protein signaling 5 exacerbates obesity, hepatic steatosis, inflammation and insulin resistance," PLoS ONE, vol. 7, no. 1, Article ID e30256, 2012.

[22] O. J. D'Cruz and F. M. Uckun, "Preclinical evaluation of a dual-acting microbicidal prodrug WHI-07 in combination with vanadocene dithiocarbamate in the female reproductive tract of rabbit, pig, and cat," Toxicologic Pathology, vol. 35, no. 7, pp. 910-927, 2007.

[23] J. M. Huston, M. Ochani, M. Rosas-Ballina et al., "Splenectomy inactivates the cholinergic antiinflammatory pathway during lethal endotoxemia and polymicrobial sepsis," Journal of Experimental Medicine, vol. 203, no. 7, pp. 1623-1628, 2006.

[24] T. R. Bernik, S. G. Friedman, M. Ochani et al., "Cholinergic antiinflammatory pathway inhibition of tumor necrosis factor during ischemia reperfusion," Journal of Vascular Surgery, vol. 36, no. 6, pp. 1231-1236, 2002.

[25] D. Altavilla, S. Guarini, A. Bitto et al., "Activation of the cholinergic anti-inflammatory pathway reduces NF- $\kappa$ B activation, blunts TNF- $\alpha$ production, and protects against splanchnic artery occlusion shock," Shock, vol. 25, no. 5, pp. 500-506, 2006.

[26] P. A. Bauerle and D. Baltmore, "NF- $\kappa$ B: ten years after," Cell, vol. 87, no. 1, pp. 13-20, 1996.

[27] M. L. Schmitz and P. A. Bauerle, "Multi step activation of NF$\kappa \mathrm{B} /$ rel transcriptional factors," Immunobiology, vol. 193, no. 24, pp. 116-127, 1995.

[28] R. Schreck, P. Rieber, and P. A. Baeuerle, "Reactive oxygen intermediates as apparently widely used messengers in the activation of the NF- $\kappa \mathrm{B}$ transcription factor and HIV-1," EMBO Journal, vol. 10, no. 8, pp. 2247-2258, 1991.

[29] F. Squadrito, D. Altavilla, B. Deodato et al., "Early activation of transcription factor NF- $\kappa \mathrm{B}$ in splanchnic artery occlusion shock," in Proceedings of the 5th World Congress on Trauma 
Shock Inflammation and Sepsis Pathophysiology Immune Consequences and Therapy, E. Faist, Ed., pp. 389-394, Monduzzi, Munich, Germany, March 2000.

[30] C. O'Mahony, "Loss of vagal anti-inflammatory effect: in vivo visualization and adoptive transfer," American Journal of Physiology, vol. 297, no. 4, pp. R1118-R1126, 2009.

[31] X. M. Song, J. G. Li, Y. L. Wang et al., "The protective effect of the cholinergic anti-inflammatory pathway against septic shock in rats," Shock, vol. 30, no. 4, pp. 468-472, 2008.

[32] K. Imai, H. Ariga, C. Chen, C. Mantyh, T. N. Pappas, and T. Takahashi, "Effects of electroacupuncture on gastric motility and heart rate variability in conscious rats," Autonomic Neuroscience: Basic and Clinical, vol. 138, no. 1-2, pp. 91-98, 2008.

[33] H. Ouyang, J. Yin, Z. Wang, P. J. Pasricha, and J. D. Z. Chen, "Electroacupuncture accelerates gastric emptying in association with changes in vagal activity," American Journal of Physiology, vol. 282, no. 2, pp. G390-G396, 2002.

[34] Q. Wang, F. Wang, X. Li et al., "Electroacupuncture pretreatment attenuates cerebral ischemic injury through $\alpha 7$ nicotinic acetylcholine receptor-mediated inhibition of high-mobility group box 1 release in rats," Journal of Neuroinflammation, vol. 9, article 24, 2012.

[35] X. Y. Gao, Y. H. Li, K. Liu et al., "Acupuncture-like stimulation at auricular point Heart evokes cardiovascular inhibition via activating the cardiac-related neurons in the nucleus tractus solitarius," Brain Research, vol. 1397, pp. 19-27, 2011.

[36] X. Y. Gao, S. P. Zhang, B. Zhu, and H. Q. Zhang, "Investigation of specificity of auricular acupuncture points in regulation of autonomic function in anesthetized rats," Autonomic Neuroscience, vol. 138, no. 1-2, pp. 50-56, 2008.

[37] I. Tekdemir, A. Asian, and A. Elhan, "A clinico-anatomic study of the auricular branch of the vagus nerve and Arnold's earcough reflex," Surgical and Radiologic Anatomy, vol. 20, no. 4, pp. 253-257, 1998.

[38] D. Gupta, S. Verma, and S. K. Vishwakarma, "Anatomic basis of Arnold's ear-cough reflex," Surgical and Radiologic Anatomy, vol. 8, no. 4, pp. 217-220, 1986.

[39] D. Engel, "The gastroauricular phenomenon and related vagus reflexes," Archiv fur Psychiatrie und Nervenkrankheiten, vol. 227, no. 3, pp. 271-277, 1979.

[40] V. A. Pavlov, H. Wang, C. J. Czura, S. G. Friedman, and K. J. Tracey, "The cholinergic anti-inflammatory pathway: a missing link in neuroimmunomodulation," Molecular Medicine, vol. 9, no. 5-8, pp. 125-134, 2003. 


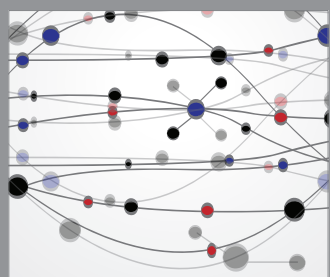

The Scientific World Journal
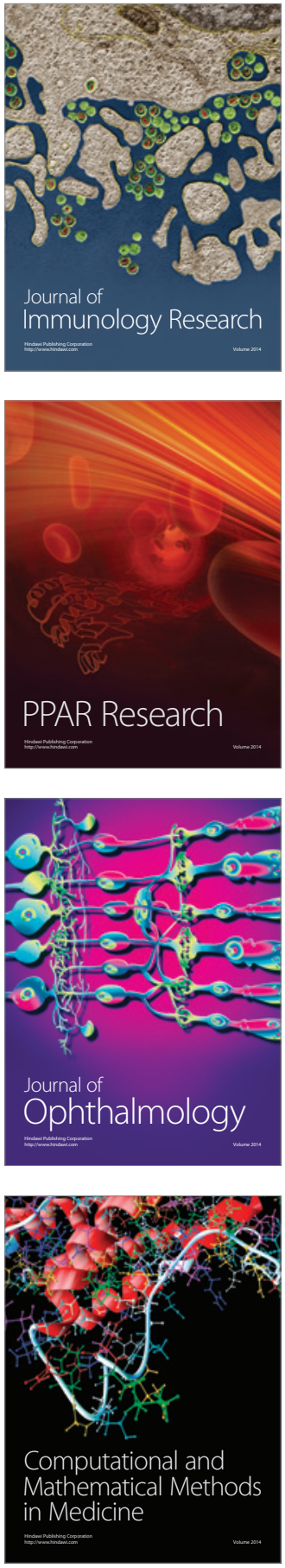

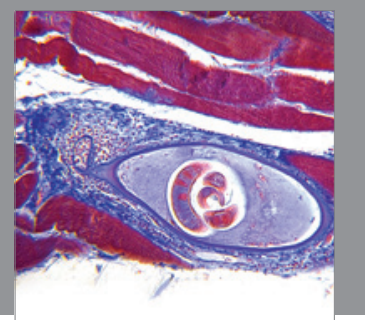

Gastroenterology

Research and Practice
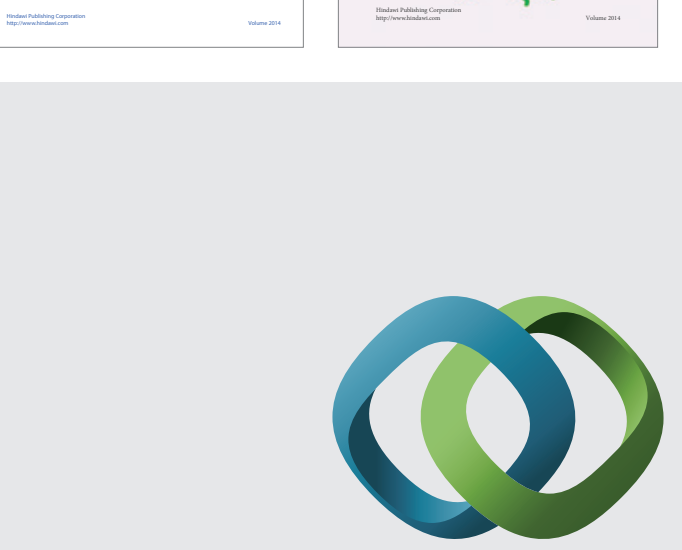

\section{Hindawi}

Submit your manuscripts at

http://www.hindawi.com
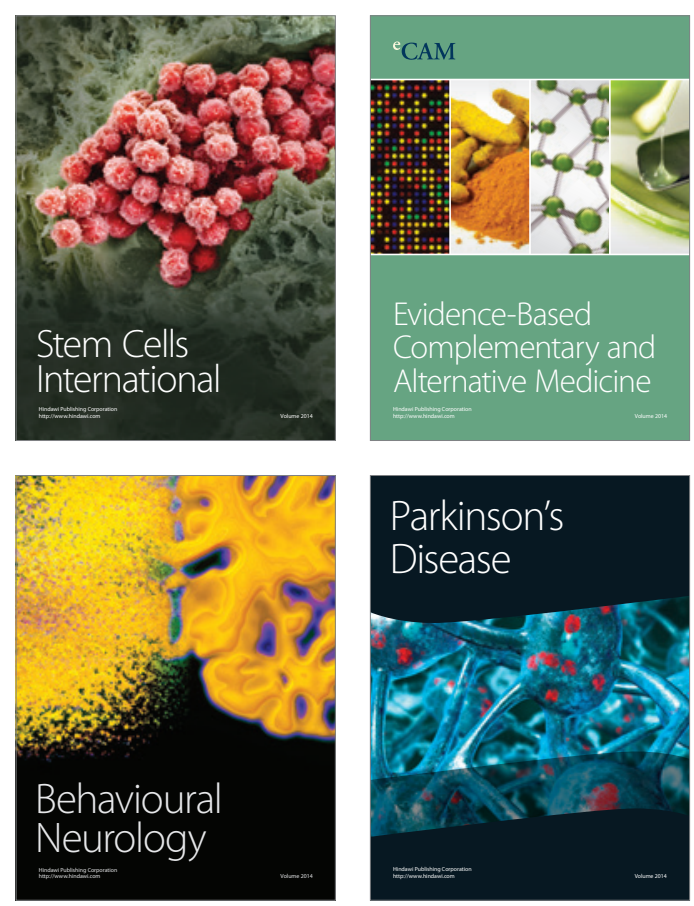

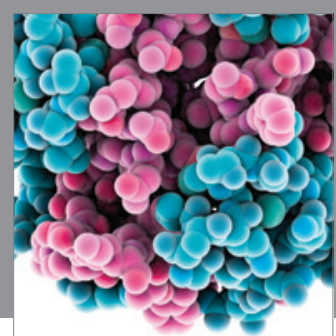

Journal of
Diabetes Research

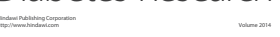

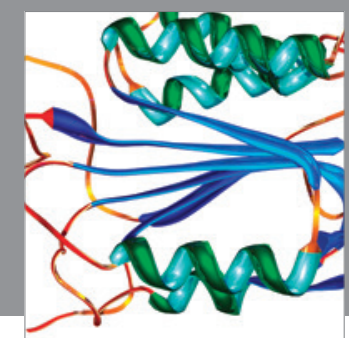

Disease Markers
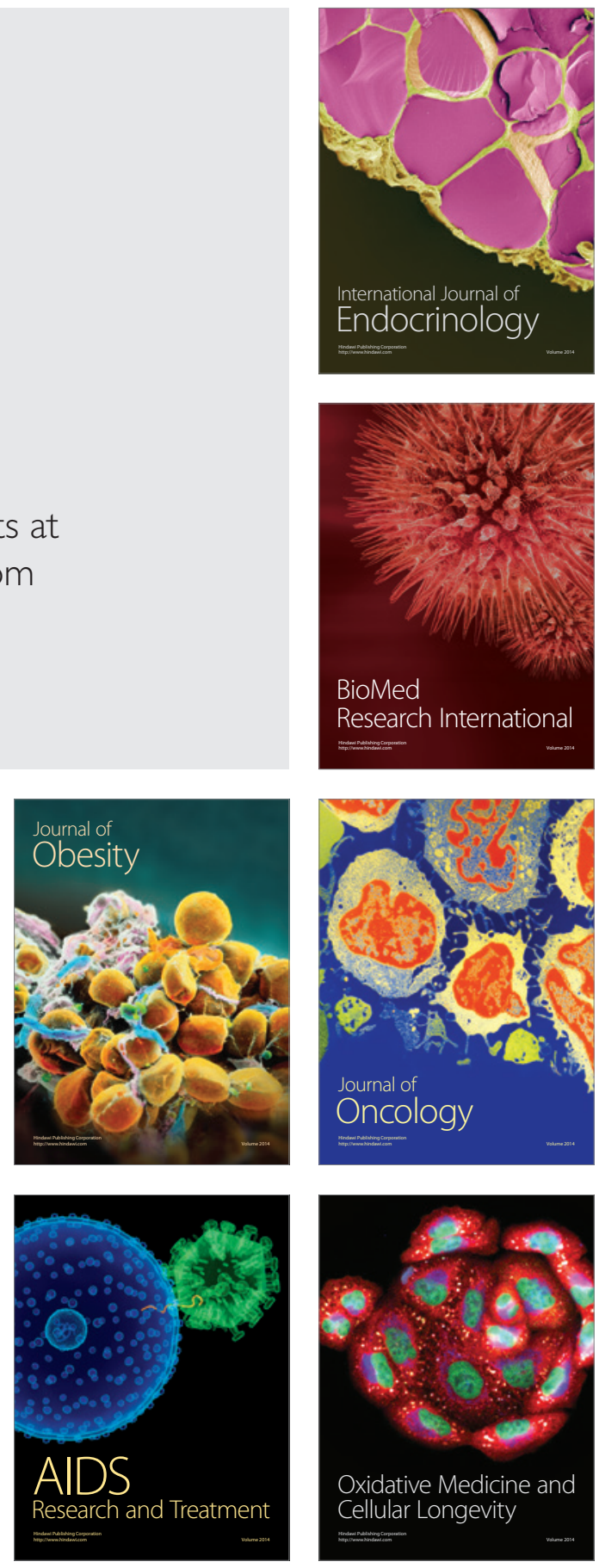